\title{
Independent Components Analysis "Artifact Correction" Distorts EEG Phase in Artifact Free Segments
}

\author{
Thatcher $\mathrm{RW}^{1 *}$, Palmero-Soler $\mathrm{E}^{1}$, North $\mathrm{DM}^{1}$ and, Otte $\mathrm{G}^{2}$ \\ ${ }^{1}$ EEG and Neurolmaging Laboratory, Applied Neuroscience Research Institute. St. Petersburg, FL, USA \\ ${ }^{2}$ Georges, Otte, Association, USA
}

*Corresponding author: Robert W Thatcher, PhD, Neurolmaging Laboratory, Applied Neuroscience Res. Inst, St. Petersburg, FL 33722, USA, Tel: (727) 244-0240; E-mail: rwthatcher@yahoo.com

Received: 31 Aug, 2020 | Accepted: 29 Sep, 2020 | Published: 06 Oct, 2020

Citation: Thatcher RW, Soler EP, North DM, Otte G (2020) Independent Components Analysis "Artifact Correction" Distorts EEG Phase in Artifact Free Segments. J Neurol Neurobiol 6(4): dx.doi.org/10.16966/2379-7150.172

Copyright: (C) Thatcher RW, et al. This is an open-access article distributed under the terms of the Creative Commons Attribution License, which permits unrestricted use, distribution, and reproduction in any medium, provided the original author and source are credited.

\section{Abstract}

EEG artifact is defined as any electrical potential that is not produced by the brain, e.g., eye movement or head movement or muscle, $50 \mathrm{~Hz}-60$ $\mathrm{Hz}$ line noise, etc. The most commonly used method of artifact elimination from an EEG recording is to delete the parts of the EEG recording that contain artifact and thereby leave the artifact free parts of the recording unchanged. Recently, Independent Components Analysis (ICA) has been used to decompose the original EEG into a set of components and then subjectively identify components that statistically load on one or more Independent Components (ICS) and using a smaller set of ICs then replace the original EEG recording with a different time series referred to as the ICA replacement or ICA-R. The purpose of this study is to mathematically and empirically test the distortion of the artifact free parts of the EEG when using ICA-R to replace the entire EEG digital record. The results of Joint-Time-Frequency-Analysis (JTFA) and the FFT spectral analyses demonstrated that ICA-Replacement of the original EEG produced phase distortions at each and every time point of the recording between all channel pairs. In contrast, the standard method of deleting the segments of an EEG recording that contain artifact did not distort the artifact free segments of the EEG recording. Conclusions are that ICA Replacement (ICA-R) is a severe distortion of the phase differences and time differences of the electrophysiology of the human scalp recorded Electroencephalogram (EEG) and invalidates all subsequent analyses that rely upon the imaginary part of the crossspectrum including scalp coherence, phase and network analyses that are dependent on the physics of electrical and magnetic fields.

Keywords: EEG; Reference; EEG phase difference; Independent components; ICA replacement; Cross-Spectrum; Invertibility

\section{Introduction}

EEG artifact is defined as any electrical potential that is not produced by the brain, e.g., eye movement or head movement, environmental sources, the TV, electric motors, etc. The accepted standards for selecting artifact free EEG were initially established by Hans Berger in the 1920s-1930s [1-3] and have been essentially unchanged since [4]. The standards were: 1-good recording "hygiene" and, 2-visual inspection to test and verify that the parts of the EEG recording used for assessment and analysis had no artifact [4-7]. Since the introduction of digital computers in the 1950s and 1960s [8-11] the standards for the selection of artifact free segments of an EEG recording have remained the same except with the addition of computational artifact detection methods based on the known physics of the EEG which arises essentially inside a sphere versus the physics of external artifacts such as heart beat, muscle, head movements, environmental noise, electrical motors, etc. that are external to the brain. Actually, external artifact sources are usually many times larger than EEG and have different frequencies and phases differences than EEG. This is why since about the 1970s most QEEG software uses the physiological artifact free parts of an EEG record to identify significant deviations and thereby delete or not select artifact for analysis [12-16]. Human scalp recorded EEG waves are unique and special and after viewing 10 or 20 examples one can recognize artifact because artifact is visually obvious and is easy to teach to recognize because external electrical sources (i.e., artifact) can be quickly deleted resulting in sufficient artifact free EEG digital samples to routinely achieve 0.9 and higher test re-test reliability. Importantly, artifact free EEG segments are the standard in clinical neurology for the evaluation of epilepsy and various pathologies [17]. This topic is also important in court cases where the invalidity of a replacement by a distortion of a patient's original digital time series with artifact can be proven.

Today computer routines are commonly used to detect and delete artifact segments or parts of an EEG recording in a few seconds and mark the EEG traces for visual verification and provide test re-test reliability statistics of the artifact free parts of the recording [12]. The vast majority of the approximately 170,000 EEG studies cited in the National Library of Medicine database (Pubmed) (6/7/20) are Quantitative EEG (QEEG) studies from clinical patients using the established method of deletion of non-brain sources of artifact by deleting artifact and then using test re-test sampling statistics of the artifact free part of the recording to test and verify that the 
selected samples are repeatable and representative of the patient's EEG recording without any replacement with an artificial substitute of the original measures.

EEG sources are hubs or clusters of synchronous synaptic potentials organized in brain networks that have different time delays between hubs (conduction velocities, synaptic rise times, synaptic delays in loops, etc.) and therefore exhibit significant and reproducible phase differences at the scalp surface [5-7,18-22]. Over the last 50 years a common practice in clinical EEG is to use a single common recording reference, e.g., an ear or mastoid or scalp electrode, etc. and then calculate the phase differences between scalp electrodes using invertible algebraic operations. Invertibility is defined by a one-to-one mapping of the domain of a function (e.g., electrode locations) to the range of a function (e.g., amplitude and phase differences, etc.). For example, if the instantaneous phase difference between scalp electrode $\mathrm{A}$ and the common reference $\mathrm{R}=60$ degrees and the instantaneous phase difference between scalp electrode $B$ and the common reference $R=45$ degrees, then the phase difference at an instant of time is (A-R)$(B-R)=A-B=60-45=15$ degree phase difference.In other words when a common reference is used in a differential amplifier, which is used in the vast majority of EEG studies cited in the National Library of Medicine database, then the phase or time differences due to the common reference electrode ' $R$ ' cancels leaving the time difference between the two active scalp leads as the physiologically accurate measure of phase difference, i.e., 15 degree instantaneous phase difference between scalp locations A and B. Also phase differences are not recoverable when using an average reference or when the recording is a bipolar montage and not a common reference $[7,17]$. Clinical Neurology and evaluations of epilepsy depend on accurate measures of phase differences as well as the direction and time delays. For example, a patient with focal left frontal lobe epilepsy that spreads or travels across the corpus callosum to the other hemisphere and/ or to other parts of the brain like the parietal lobe is part or the clinical presentation. Therefore, phase difference in a patient's EEG is important information in the evaluation of a patient with epilepsy and other neurological disorders.

The property of invertibility is important because it allows for inverse solutions of the 3-dimensional sources of the EEG and based on spatial information related to phase or time differences between separated groups of neurons. This is why a linear filter called Finite Impulse Response (FIR) is used in EEG because of the preservation of phase or time differences in the EEG recording. Infinite Impulse Response filters (IIR) fail to preserve phase and extra steps are needed to recover phase differences with an IIR filter. The mathematics of invertibility allows for algebraic remontaging that can be reversed with no loss of information and there is no distortion of the phase differences present in the original recording $[4,6,7,19]$.

Accurate measures of the scalp electrical potentials and phase differences depends on the physics of the EEG and the use of invertible equations such as the Poisson, Laplace, Green's equations and Maxwell and Helmholtz equations. Once the mapping of the domain to the range of a function is artificially modified or if nonlinear filtering is used then error is introduced into the equations of physics and also there is a decoupling of the brain from EEG network measures [7,12,23-26]. Unfortunately, recent developments in decomposing the EEG into Independent Components (ICs) and then creating a replacement time series using a smaller set of ICs is a nonlinear filter process that is not invertible and therefore has created an alternate reality by replacing the original voltages by a mathematical construct that lacks mathematical and physiological validity.
Independent Components Analysis (ICA) is a solution to the "cocktail party problem" of listening in on one person's speech in a noisy room. If $\mathrm{N}$ voices (sources) are present, at least $\mathrm{M}$ microphones (observations) are needed to recover the original signals. This constitutes the square case or $\mathrm{M}=\mathrm{N}$ that is "invertible" or where one can uniquely determine the input from its output. If there are more sources (neurons) then sensors (microphones) or where $\mathrm{M} \neq \mathrm{N}$ then the system is non-invertible. Also, Independent Components Analysis (ICA) is based on three non-physiological assumptions:

\section{1- $\quad$ The sources are independent \\ 2- The sources are non-Gaussian in distribution and, \\ 3- The sources are spatially fixed.}

The number of EEG recording electrodes (microphones) is many times smaller than the number of sources. In addition, ICA assumes that the sources (voices) are independent but neurons are organized in clusters or hubs in networks with reciprocal loops and are not independent. Neurons fire with bursts of action potentials and communicate with distant clusters of synchronous neurons by axons and synapse connections with conduction velocities and synaptic delays as a function of the distance between hubs and coordinating structures like the thalamus, cortico-cortical connections, etc. This means that when decomposing the EEG time series into a Set of Independent Components (ICS), then the ICs are not sources of the EEG and cannot be used as a replacement of the EEG electrical field of the brain nor to accurately estimate the inverse solution to link 3-dimensional sources inside of the brain to the 2-dimensional scalp electrical field. Instead ICs are mathematical constructs that decompose the electrical field using a noninvertible and nonlinear method that is also physiologically invalid. Another violation of the ICA model is the fact that all of the generators of the human EEG are not non-gaussian.

Simple transforms such as log base 10 and Box Cox or square root are commonly used in QEEG analyses $[4,12,13,27,28]$ to produce Gaussian distributions and many metrics are naturally Gaussian.

Figure 1 is an example of an approximately Gaussian distribution of phase differences and simple transforms are used to better approximate Gaussian if necessary [12,13,29-31] [Figure 1].

ICA is invertible when the number of independent components equals the number of scalp electrodes or where there is a one-to-one mapping of the domain (ICs) to the range (loadings). However, ICA is not invertible when it is used to reconstruct a replacement time series (ICA-R) from a smaller domain than the original domain and there is no longer a one-to-one mapping. The ICA artifact correction thought to remove artifact such as eye movement and blinks is an example of a non-invertible process. ICA reconstruction of a substitute noninvertible time series is by a three step process:

1- $\quad 1^{\text {st }}$ decompose $\mathrm{N}$ scalp EEG recordings into N ICs,

2- Eliminate one or more ICs that subjectively appear to have high artifact loadings and,

3- Then with the smaller set of ICs create a substitute ICA-R time series that replaces the original EEG. The 19 channel ICA-R time series replacement of the original EEG recording invalidates all subsequent phase dependent analyses because of the violation of the property of invertibility. In the 1800s Bernard Reimann solved and defined the universal invertibility properties that analytic functions depend on such as Fourier and Hilbert transforms as well as the inverse function theorem and matrix algebra in general. 


\section{Example of an Approximate Gaussian Distribution of EEG Phase Differences with No Transforms}

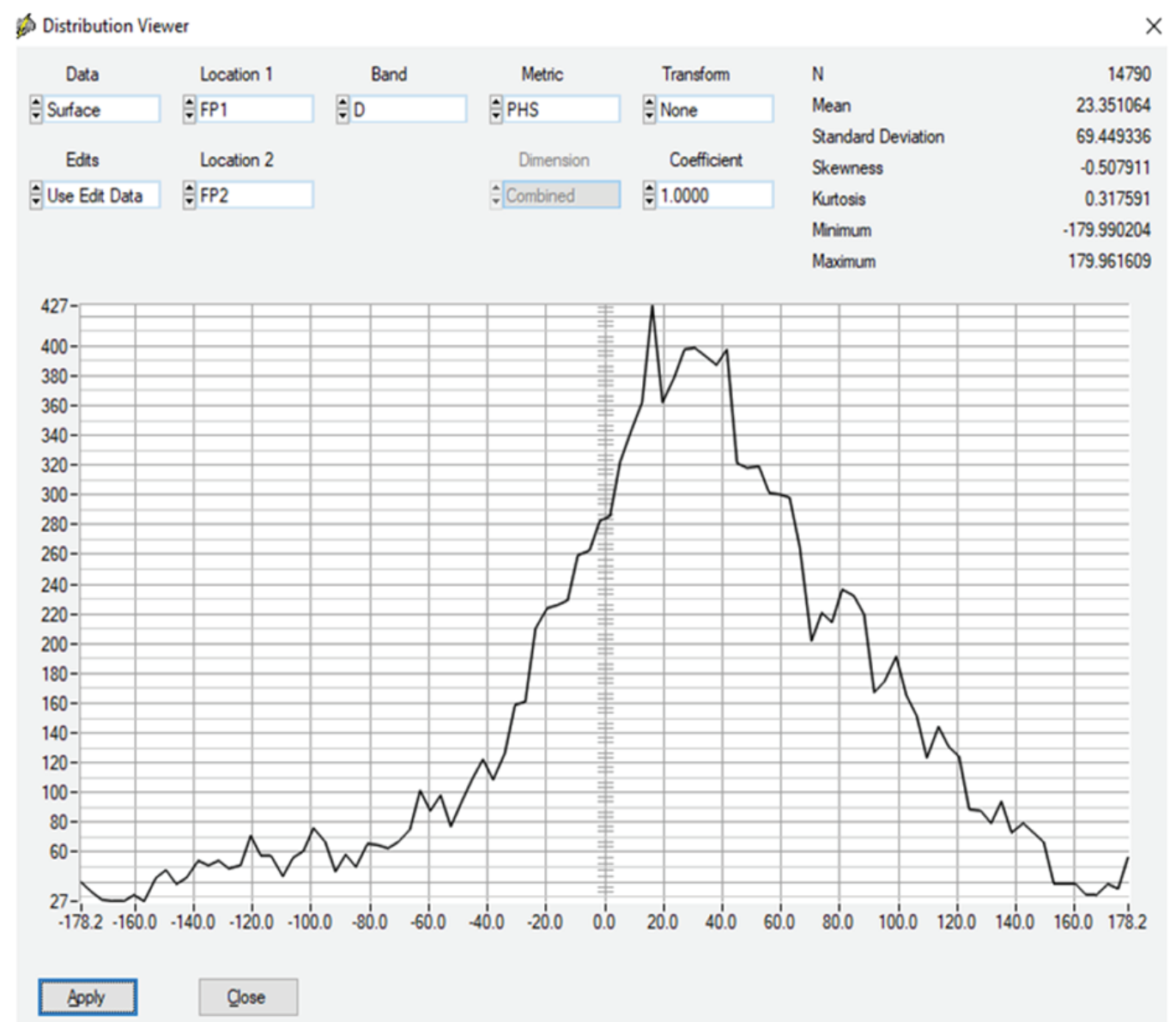

Figure 1: An example of an approximate Gaussian distribution of EEG phase differences. In this instance the phase differences are between the $\mathrm{Fp} 1$ and $\mathrm{Fp} 2$ scalp locations in the delta band for 2 minutes of artifact free EEG selections. Very similar Gaussian distributions are present for other electrode combinations and different frequency bands.

Figure 2 illustrates the difference between invertible linear relationships (top) $v s$. the ICA non-invertible relationship after one or more ICs (range) are removed (bottom).

At first glance ICA reconstruction sounds promising but has a serious limitation because the replacement ICA-R time series has altered all of the amplitude and phase differences of the original time series, including the artifact free segments and there is no inverse transform that maps the original time series to the lower number of ICs. For example, Montefusco-Siegmund R, et al. [23] concluded that "in this work we showed that ICA artifact correction would introduce non-linear and non-stationary phase changes over time and across frequencies, setting spurious phase coherence indexes." Therefore, the assumptions that underlay ICA fail to fit the physiological reality of the human EEG. Because of the violation of the physiological reality of the EEG, Hyvainen A, et al [32] stated: "It should be noted that this technique [ICA] may lead to the insertion of undesirable new artifacts into the brain recordings (Jervis BW, et al. 1989)" [33]. Castellanos NP and Makarov VA [26] concluded: "a separation is valid for independent, linearly mixed sources when their total number does not exceed the number of recording electrodes (Bell AJ and Sejnowski TJ [34]). In practice these assumptions can be violated leading to a "leak" of the cerebral activity into components deemed artificial. Complete rejection of such a component supposes a partial loss of the neural signal."

Some justify the use of ICA reconstruction by wrongly believing that all EEG recordings contain artifact and therefore there is no ground truth of the EEG. This justification for the use of ICA reconstruction is invalid as attested to by the fact that there are over 170,000 peer reviewed EEG studies cited in the National Library of Medicine database (Pubmed, 2020) with high consistency and high test retest reliability and high cross-validation to various clinical conditions because the artifact free segments of the EEG without the use of ICA were used in these studies. A search of the National Library of Medicine database (Pubmed) using the search terms: "ICA rejection of EEG artifact" yields only 52 citations. If all EEG was contaminated by artifact as the supporters of ICA replacement claim then this literature would not exist nor have high reliability and validity of the EEG [4$14,35,17,27]$. To appreciate the difference of the process, consider this situation: if a recording of 3 minutes contains only one blink artefact of 1 second the ICA-R method will apply (and change) the complete recording ( 3 minutes) including the artefact free segments in contrast with the traditional artefact removal techniques where only the 1 

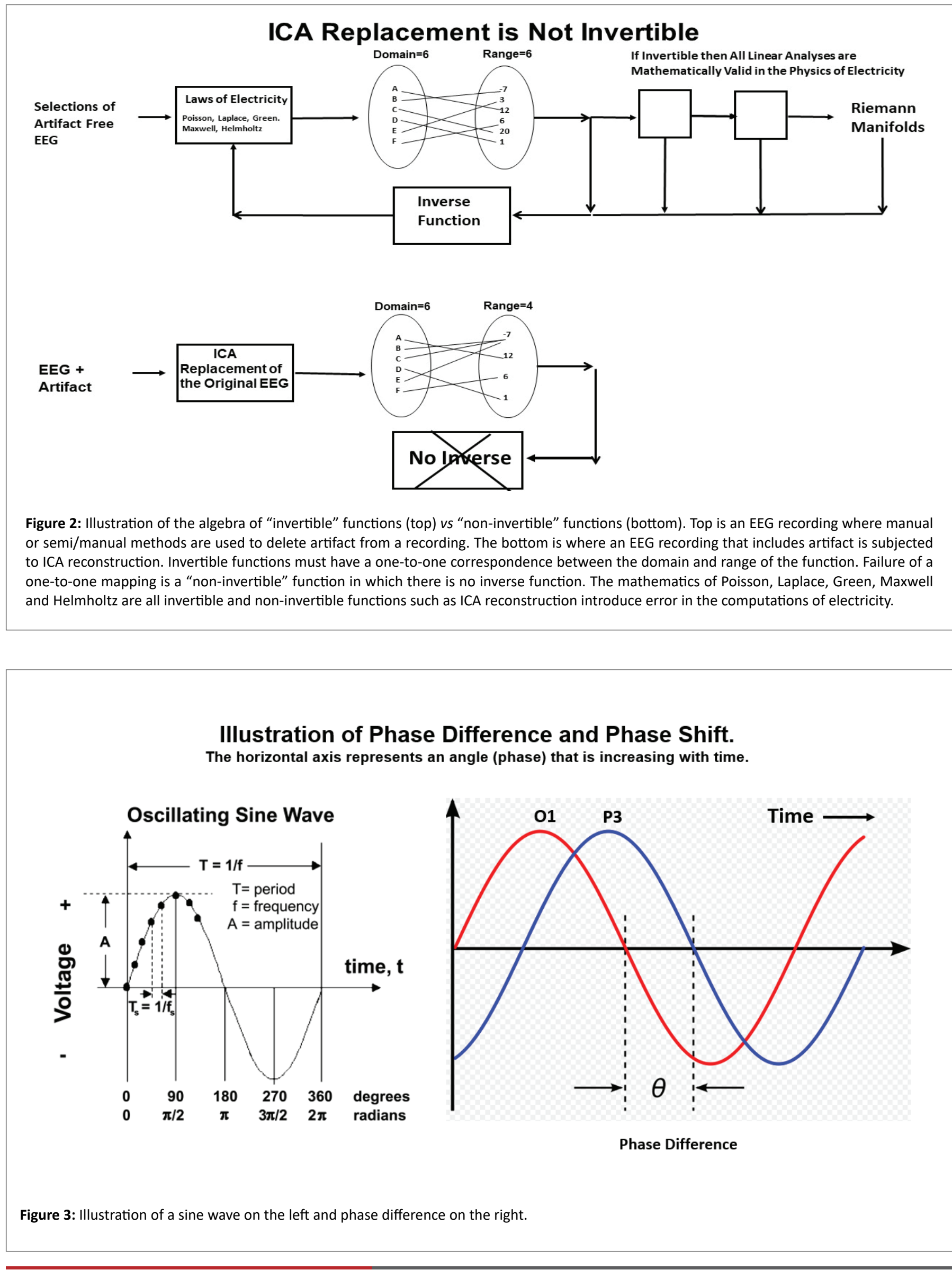

Citation: Thatcher RW, Soler EP, North DM, Otte G (2020) Independent Components Analysis "Artifact Correction" Distorts EEG Phase in Artifact Free Segments. J Neurol Neurobiol 6(4): dx.doi.org/10.16966/2379-7150.172 
second artefact containing segment is removed leaving the rest of the original recording unaltered. ICA-R is a very incisive technique that replaces any original signal by a digital reconstruction.

The goal of this study is to provide pre $v s$. post empirical evidence that Independent Component Analysis (ICA) to reconstruct a substitute time series that visually appears to remove artifact but actually creates artifact and distorts phase differences present in the artifact free parts of the original time series. To counter the argument that all EEG recordings contain artifact we will show that phase distortion occurs for each and every time sample for all combinations of electrodes including the artifact free portions of the EEG. Also, we cite the scientific literature demonstrating high test re-test reliability of EEG as well as high clinical effect sizes which would not exist if EEG was always contaminated by artifact $[7,35,27]$.

\section{Methods}

\section{Subjects and EEG recordings}

A total of 11 non-epileptic clinical subjects ranging in age from 18 to 33 years ( 8 males) were included in this study. The EEG was recorded using a Mitsar amplifier model 202 at a sample rate of 250 $\mathrm{Hz}$ in the eyes open condition. Three to seven minutes of EEG of 19 channels of EEG was recorded according to the 10/20 electrode locations referenced to linked ears.

\section{Manual artifact free selections $v s$. ICA reconstruction}

Visual inspection and manual deletion of eye movement and blink and any artifact was conducted and the artifact free original EEG was saved in an EDF formatted file. In addition, WinEEG software (Version 2.11) was used to compute blind source separation using Independent Component Analysis (ICA) based on the Infomax algorithm [36,37]. After the decomposition of the 19 channel EEG the component related to eye movement artifacts were manually selected and after removing the eye movement related Independent Component (IC) then based on the remaining 18 Independent Components (ICs) a replacement 19 channel time series was computed. As mentioned previously, this is referred to as ICA replacement or reconstruction and the new replacement 19 channel time series is referred to as the ICA-R time series. The ICA-R data were also saved in EDF format. To verify that the WinEEG ICA-R was computed correctly the original EEG and the WinEEG ICA-R data from a single subject (subject \#11 duration of 6 minutes \& 51 seconds) was sent to experts at the Univ. of California at San Diego (UCSD) and to the Eeglablist for others to independently evaluate the pre $v s$. post ICA data. Individuals on the EEG Lab list used standard software $[37,38]$ to perform ICA-R where two independent components representing eye movement and blink artifact were deleted and the remaining 17 Independent Components (ICs) were used to reconstruct a replacement 19 channel time series (ICA-R). This provided a further independent validation of phase difference distortion and also compared the WinEEG ICA-R to the UCSD ICA-R using the same original EEG data.

\section{Spectral analyses}

The Hilbert transform was used to compute the "instantaneous" auto and cross-spectrum for each time sample and for all combinations of electrodes for the hand edited original EEG data as well as the ICA-R, time series for each subject. Instantaneous coherence and phase differences were calculated for all channel combinations for four different frequency bands (delta 1-3.99 Hz; theta 4-7.99 Hz; alpha $8-12.99 \mathrm{~Hz}$; beta $13-30 \mathrm{~Hz}$ ). The time points selected by the hand editing method were saved and also used for an FFT analysis of the
ICA-R data. In this way the exact same time samples were used for both the original 19 channel time series and the 19 channel ICA-R time series. Statistical analysis involved first comparing the percent difference between the original hand edited EEG and the ICA-R time series and then paired t-tests were computed between the original hand edited EEG and the ICA-R time series.

\section{Calculation of phase differences}

Phase is the position of a point in time (an instant) on a waveform cycle. A complete cycle is defined as the interval required for the waveform to return to its arbitrary initial value. Phase difference is the difference, expressed in radians, degrees or time, between two waves having the same frequency and referenced to the same point in time. Two oscillators that have the same frequency and different phases have a phase difference and the oscillators are said to be out of phase with each other. The amount by which such oscillators are out of phase with each other can be expressed in degrees from $0^{\circ}$ to $360^{\circ}$, or in radians from 0 to $2 \pi$. If the phase difference is 180 degrees ( $\pi$ radians), then the two oscillators are said to be in antiphase.

Phase shift is any change that occurs in the phase of one quantity, or in the phase difference between two or more quantities. The symbol is sometimes referred to as a phase shift or phase offset because it represents a "shift" from zero phase difference. For infinitely long sinusoids, a change in is the same as a shift in time, such as a time delay. If $x(t)$ is delayed (time-shifted) by $1 / 4$ of its cycle, it becomes:

$$
x\left(\frac{1}{4} T\right)=A \cos \left(2 \pi f\left(t-\frac{1}{4} T\right)+\varphi\right)=A \cos \left(2 \pi f t-\frac{\pi}{2}+\varphi\right)
$$

Where "phase" is now $\varphi-\frac{\pi}{2}$ and has been shifted by radians (the variable $\mathrm{A}^{\circ}$ represents the amplitude of the wave).

Figure 3 is an illustration of phase difference:

A common method to compute phase differences is by first computing the power spectra for $\mathrm{x}$ and $\mathrm{y}$ (i.e., two different EEG channels) and then the normalized cross-spectra. Since complex analyses are involved this produces the cospectrum (' $r$ ' for real) and quadspectrum (' $\mathrm{q}$ ' for imaginary). The phase difference between $\mathrm{x}$ and $\mathrm{y}$ is computed as: phase $=159.1549 \tan ^{-1}(\mathrm{q} / \mathrm{r}) / \mathrm{f}$ where $\mathrm{f}$ is the center frequency of the Hilbert transform. In complex numbers coherence is the normalized vector length $C o h=\sqrt{r^{2}+q^{2}}$ phase $\Xi_{x y}=\alpha$ is the angle represented by the arc tangent of $\mathrm{q} / \mathrm{r}$ and $\mathrm{SC}$ is the center frequency.

$$
E Q(2) \Xi_{X Y}=\left(\operatorname{Tan}^{-1} \frac{q_{x y}^{2}}{r_{x y}^{2}}\right) / S C
$$

Phase difference is represented by radians or degrees but also in milliseconds when the tangent is multiplied by 159.1549 or phase difference $=159.159 \tan ^{-1}(q / r) / S C \quad[7,12,19,21]$. The average phased difference for a given electrode spacing and frequency is often computed using the FFT. Mathematically the cross-spectrum between a pair of channels at one frequency is defined as the product of the signal from one channel with the complex conjugate of the signal of the other channel. This produces a two-dimensional vector on the complex plane where the orientation of the vector is equal to the difference of orientations of the phases of the two signal vectors is the measure of EEG phase difference between the two signals. 
The Hilbert transform is a Joint-Time-Frequency-Analysis (JTFA) that is used to compute phase differences for a given frequency for each and every time sample and is called instantaneous phase differences. The analytical signal associated with the time series $x_{1}(t) i s Z_{1}(t)=a_{1}(f) e^{j \phi 1(t)}$ where the imaginary part $y_{1}(t)=\operatorname{Im}\left\{Z_{1}(t)\right.$ is the Hilbert transform of the signal $x_{1}(t)$ The signal is bandpass filtered around the frequency of interest and then the instantaneous phase is estimated from the analytical signal as: $\Phi x_{1}(t)=\arg \left\{x_{1}(t)+j_{1}(t)\right\}$ The phase difference between two EEG channels $x_{1}(t)$ and $x^{2}(t) i s \phi 1, x^{2}(t)=m \phi x 1(t)-n \phi x^{2}(t)$. One may compute the $1^{\text {st }}$ derivative of phase differences and if the phase differences are constant over time then this is called "Phase Locking". Phase lock duration is the period of time between phase shifts or the period of time when the $1^{\text {st }}$ derivative approximates 0 that begins and ends when the $1^{\text {st }}$ derivative is a large absolute value greater than some criteria value called a phase shift $[14-16,39,40]$.

Another expression of phase difference is by Bendat JS and Piersol AG [35] where phase difference is a function of frequency, distance and conduction velocity: i.e.,

$$
\mathrm{EQ}(3) \Theta_{x y}=\frac{2 \pi f d}{c}
$$

Where $\Xi_{X Y}$ is the phase lead or lag between locations $\mathrm{x}$ and $\mathrm{y}$, $\mathrm{f}$ is the frequency, $\mathrm{d}$ is the distance traveled in meters and $\mathrm{c}$ is the conduction velocity in $\mathrm{m} / \mathrm{sec}$ [35].

As mentioned previously in the brain the physiological ground truth of EEG phase or time differences are based on the fact that neurons are connected in loops and between network hubs with accumulated delays both within the neocortex and between cortical and subcortical structures. The ground truth of EEG phase differences between separated scalp locations are physiological factors of the physics of electrical sources and the connections between sources (i.e., networks) such as axonal conduction velocities, length of connecting axons, synaptic rise times, synaptic delays and synaptic integration times, etc. $[4,7,18-20]$.

\section{Results}

\section{Evidence that ICA reconstruction and EEG replacement alters the phase differences of the EEG}

The ICA-R reconstruction performed by the UCSD group for one subject were very similar to those performed by the WinEEG software and showed essentially the same differences although there were fewer significant differences using the USCD ICA. Because of space limitations, the UCSD ICA-R method [37] which is a standard and widely used ICA method will be presented as a representative example of the same differences between the original EEG and the ICA-R time series in all of the subjects.

The Hilbert transform confirmed that the phase differences had been altered for each and every time sample and for all electrode combinations in the artifact free sections of the EEG recording. Figure 4 is an example the absolute phase differences in degrees between the O1 channel and the other 18 channels using the Hilbert transform. The left panel is the phase differences in the original recording and the right panel are the phase differences after ICA reconstruction. Visual inspection shows that phase differences are not the same between the left and right panels. The vertical line at 12 seconds and 400 milliseconds from the beginning marks the phase difference at an instant of time between the original EEG recording and the ICA reconstructed time series. The panel of numbers to the right of the tracings is the exact phase differences in degrees for all channel combinations at this instant of time. There was no artifact in this part of the recording and one can demonstrate that phase differences have been altered for each and every time point and all channel combinations and all frequency bands for the entire record. Figures 5 and 6 are additional examples of phase distortion in the artifact free parts of the recording.

Figure 5 is another example of differences in the absolute phase differences in degrees between the T5 channel and the other 18 channels using the Hilbert transform at a different instance of time in the recording where again there was no artifact (1 minute and 21 seconds after the start of the recording). The left panel is the phase differences in the original recording and the right panel are the phase differences after ICA reconstruction. Visual inspection shows that phase differences are not the same between the left and right panels. The panel of numbers to the right of the tracings is the exact phase differences in degrees for all channel combinations at this instant of time.

Another example of alterations in instantaneous phase differences in artifact free parts of the EEG recording is shown in figure 6 . The absolute phase differences in degrees are between the P4 channel and the other 18 channels using the Hilbert transform at a different instance of time in the recording where again there was no artifact (3 minutes and 4 seconds after the start of the recording). The left panel is the phase differences in the original recording and the right panel are the phase differences after ICA reconstruction. Visual inspection shows that phase differences are not the same between the left and right panels. The panel of numbers to the right of the tracings is the exact phase differences in degrees for all channel combinations at this instant of time.

\section{Alteration of Phase Differences by ICA Replacement (ICA-R) Using the FFT}

EEG analyses of brain networks requires using measures such as phase delays, coherence, lagged coherence, phase slope index, phase lock, phase shift, phase-amplitude coupling, cross-frequency coupling and directed coherence to name a few commonly used methods. The scientific literature on this range of network measures is large and beyond the scope of this paper to review. However, all of these measures of network dynamics depend on accurate and reliable measures of the electrical phase differences in the original recording and if phase differences between channels in the original EEG recording are irreversibly altered using an invalid method then all subsequent network analyses are invalid.

Statistically significant differences between the phase differences in the original EEG recording vs. post ICA-R were present in all eleven subjects. Table 1 show that the percentage of statistically significant comparisons $(\mathrm{P}<0.05)$ for 171 pairs of channels ranged from $13.5 \%$ to $98.2 \%$.

Figure 7 is an example of the percent difference in phase differences between channels for the original pre-ICA record $v s$. the Eeglablist (UCSD) post ICA-reconstructed time series (ICA-R time series).

Figure 8 are the results of paired t-tests between the same $1 \mathrm{~min}$ \& $42 \mathrm{sec}$ of artifact free parts of the a patient's original EEG phase differences $v s$. the same time points in post ICA-R' reconstructed phase differences conducted by the UCSD Eeglablist method where $87.5 \%$ were statistically significant $(\mathrm{P}<0.05)$. 


\section{Comparison of Instantaneous Phase Differences Between Non-ICA vs ICA Reconstructed Phase Difference Between 01 vs Remaining Channels}

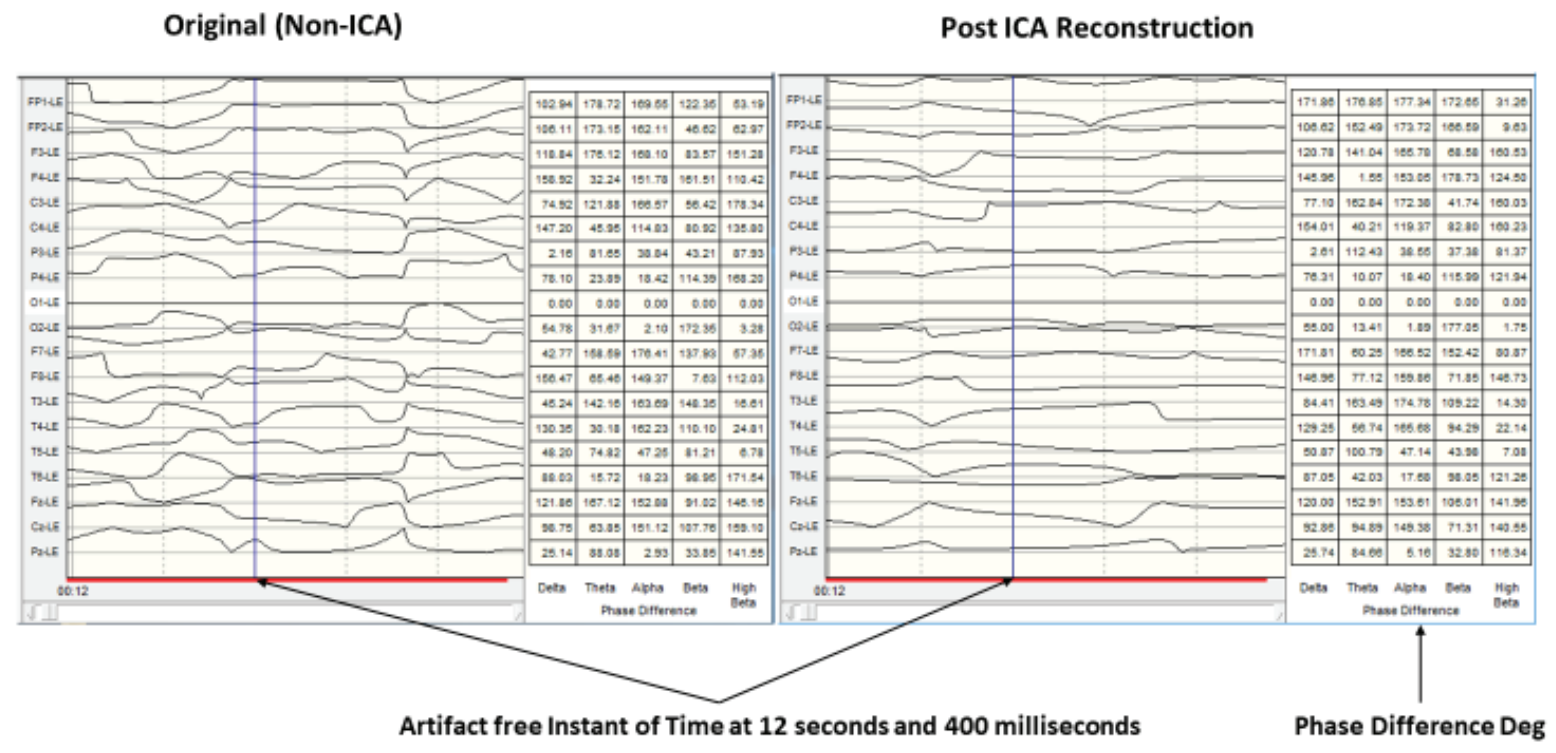

Figure 4: The columns on the left are EEG channel labels and the columns on the right are absolute phase differences in degrees for the four frequency bands. The "Original (Non-ICA)" image are absolute phase differences in a patient with attention deficit disorder with respect to 01 and the other 18 channels in the original EEG recording and the right "ICA Reconstruction-(UCSD EEG Lab)" image are the phase differences after the ICA reconstruction for the exact same time points and channel combinations. The box to the right of the phase difference tracings are the phase difference values (degrees) at exactly 12 seconds and 400 milliseconds from the start of the recording. This part of the EEG recording was artifact free. A careful comparison demonstrates that the phase differences have been altered in each and every artifact free time sample for the entire EEG record and for all frequencies and all channel combinations.

The same large changes in other network metrics have been evaluated for numerous subjects. Because of page limitations it is not possible to present all of these analyses. Suffice it to say that all of the network analyses demonstrated similar magnitude of difference between the original artifact free parts of a recording and the post ICA replacement time series. A You Tube video demonstration of the alteration of phase differences by ICA-R replacement is at: http:// youtu.be/BfqCh2UeJik

\section{Discussion}

The results of this study support the conclusions of numerous distinguished scientists and the Montefusco-Siegmund R, et al. [23] simulation analyses that: "in this work we showed that ICA artifact correction would introduce non-linear and non-stationary phase changes over time and across frequencies, setting spurious phase coherence indexes." The findings in this study also support the cautionary words of Mannan MMN, et al. [24]: "Although the performance of ICA is promising, it should be employed with care [25]. Also Castellanos NP and Mkarov VA [26] stated that "the artifact suppression may also corrupt the power spectrum of the underlying neural activity." and that "Rejection of such components supposes a loss of a part of the cerebral activity and, consequently, distortion of the artifact free EEG.

Hyvarinen A, et al. [32] stated: "In noisy ICA, we also encounter a new problem: estimation of the noise-free realizations of the independent components (ICs). The noisy model is not invertible" [32], Hyvarinen A, et al. [32] further state: "A difficult problem in independent component analysis (ICA) is encountered if the number of mixtures $x i$ is smaller than the number of independent components. This means that the mixing system is not invertible" [32]. "Thus, the mixing matrix has size $\mathrm{m} \times \mathrm{n}$ with $\mathrm{n}>\mathrm{m}$, and therefore it is not invertible." [32]. Wallstrom G, et al. [42] concluded: "We were disappointed by the performance of ICA for artifact correction, both in our simulation study and in investigations with real data." and that: "studies are needed to further investigate the potential spectral distortions induced by ICA".

Finally, the results of the present study are consistent with statements in 2014-2017 by experts on ICA at the University of California at San Diego and elsewhere:

- "If you remove IC and reconstruct channel EEG by back projecting the remaining ICs, of course it changes channel EEG phase!" (Makoto Miyakoshi, Eeglablist ICA and signal phase content, Sept. 16, 2014)

- "The EEG reconstruction after removing bad components/sources MAY change the phase value of the signal at any electrode." (M. Rezazadeh Eeglablist ICA and signal phase content, Sept. 18, 2014).

- "The reconstructed data after removing spurious ICA components differs from the original time series, and because 


\section{Comparison of Instantaneous Phase Differences Between Non-ICA vs ICA Reconstructed \\ Phase Difference Between T5 vs Remaining Channels}

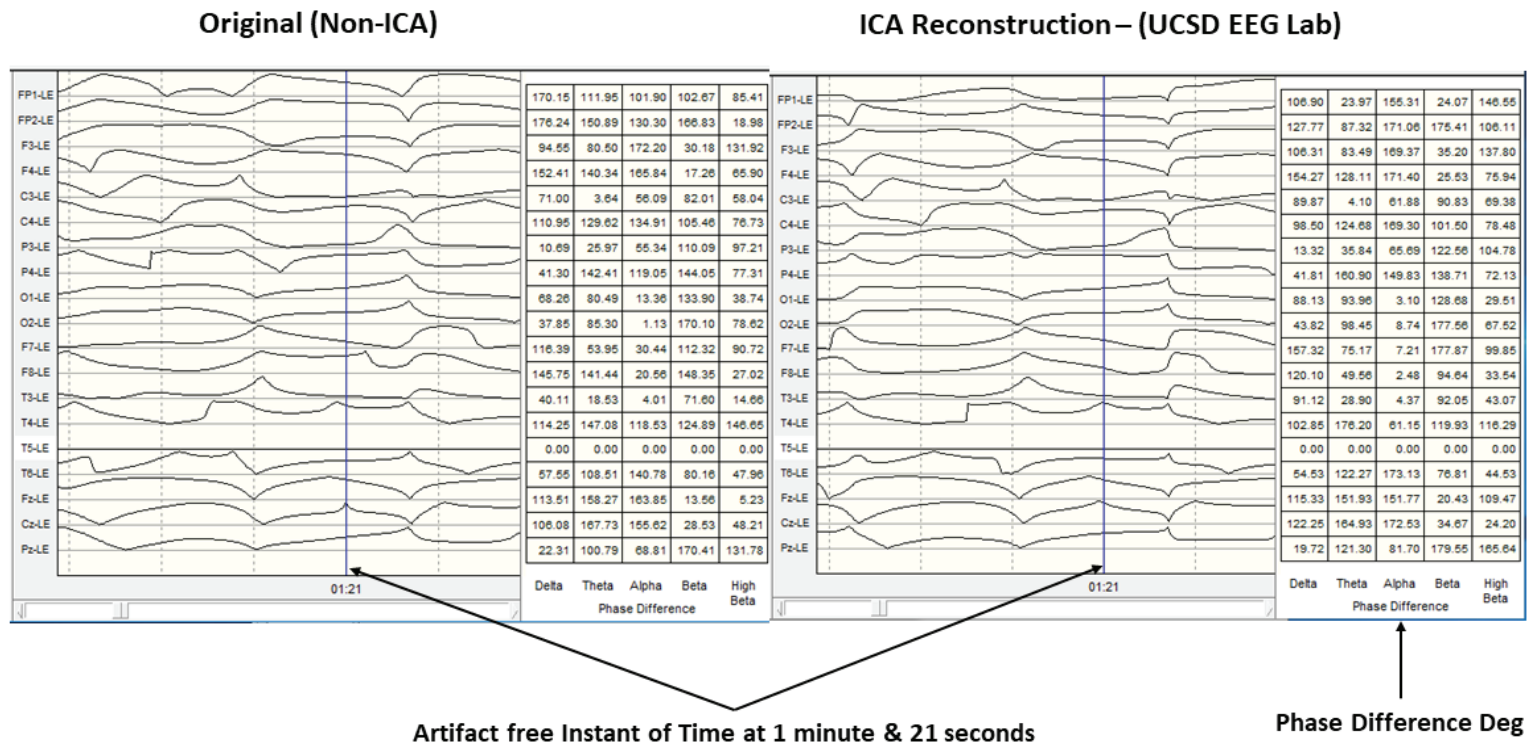

Figure 5: The columns on the left are EEG channel labels and the columns on the right are absolute phase differences in degrees for the four frequency bands. The "Original (Non-ICA)" image are absolute phase differences in a patient with attention deficit disorder with respect to T5 and the other 18 channels in the original EEG recording and the right "ICA Reconstruction-(UCSD EEG Lab)" image are the phase differences after the ICA reconstruction for the exact same time points and channel combinations. The image on the right are the phase differences after the ICA reconstruction for the exact same time points and channel combinations. The box to the right of the phase difference tracings are the phase difference values (degrees) at exactly 1 minute and 21 seconds from the start of the recording. This part of the EEG recording was artifact free. A careful comparison demonstrates that the phase differences have been altered in each and every artifact free time sample for the entire EEG record and for all frequencies and all channel combinations.

of that there are phase differences." (Arnaud Delorme, Eeglablist ICA misinformation, June 10, 2017).

- "I first noticed the problem with phase distortion more than a decade ago" (Robert Lawson, Eeglablist ICA misinformation, June 14, 2017).

- "I think he is right that the relative phase will be changed by deleting 1 or 2 artifact components." (Ramesh Srinivasan, Eeglablist ICA misinformation, June 14, 2017).

- "We found phase distortions in the $8-10 \mathrm{~Hz}$ alfa band (greatest near the source of artefact) but also on more remote electrodes such as occipital and also in artefact free strokes of EEG." (Georges Otte, Eeglablist ICA misinformation, June 15, 2017).

In some situations phase differences are irrelevant, for example, when measuring AM radio waves. In other situations phase differences are critical, for example, TV signals. In evoked potential analyses phase differences are not considered important because of the assumption that all of EEG is "volume conduction" that is defined as phase difference equals zero. However, in EEG network analyses phase differences are critical, for example, coupling strength or the direction and magnitude of information flow or conduction velocities, etc. ICA replacement is like an IIR filter that alters both amplitude and distorts phase. If one uses a FIR filter or a zero-phase-shift digital filter then there is no phase distortion. Therefore, the answer to the question "does filtering make things worse?" is YES especially when each and every time sample is distorted in an entire EEG recording that does not contain artifact and the transform is non-invertible.

There are two main categories of artifact correction of the EEG:

1- Deletion of the parts of the recording that contain artifact without any alteration of the artifact free portions of the recording and,

2- Mathematical decomposition and then replacement of a non-invertible time series that has altered all of the values of the original time series including the artifact free parts of the recording. The recording reference is important in the first category but is irrelevant in the second category because the alteration of the time samples in the original recording decouples the subject's brain from the underlying ground truth physics of the EEG. ICA replacement replaces the original time series with artifact and once alteration of data at the primary level occurs then all subsequent network analyses, including re-referencing are invalid. While amplitude alteration by ICA replacement may have little impact on event related potentials the situation with spontaneous EEG is more serious. More significant is the lack of justification for any alteration at the primary level of data in the first place.

Over 120,000 peer reviewed quantitative EEG studies have been published since the late 1950s that use standard artifact deletion methods without any alteration in the artifact free parts of the EEG 


\section{Comparison of Instantaneous Phase Differences Between Non-ICA vs ICA Reconstructed Phase Difference Between P4 vs Remaining Channels}

\section{Original (Non-ICA)}

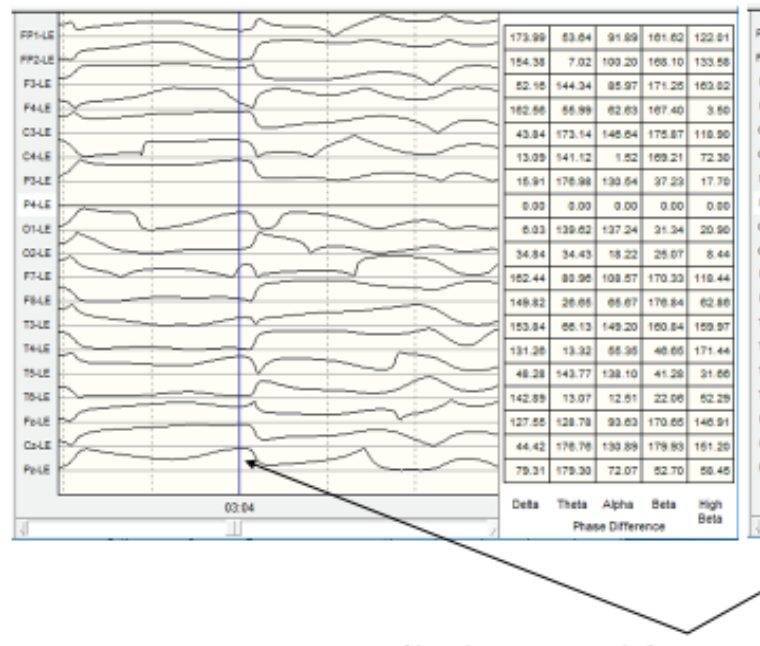

Artifact free Instant of Time at 3 minutes \& 4 seconds
Post ICA Reconstruction

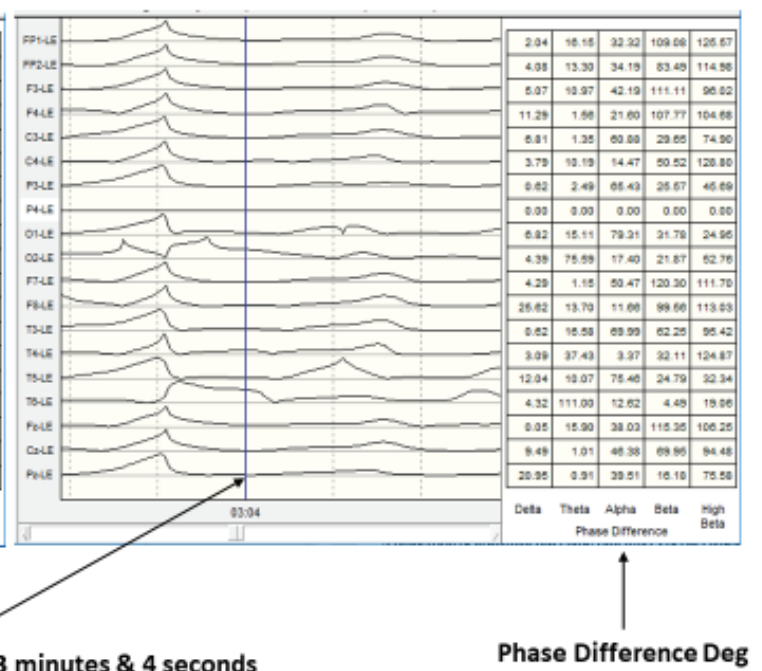

Figure 6: The columns on the left are EEG channel labels and the columns on the right are absolute phase differences in degrees for the four frequency bands. The "Original (Non-ICA)" image are absolute phase differences in a patient with attention deficit disorder with respect to P4 and the other 18 channels in the original EEG recording and the right "ICA Reconstruction-(UCSD EEG Lab)" image are the phase differences after the ICA reconstruction for the exact same time points and channel combinations. The image on the right are the phase differences after the ICA reconstruction for the exact same time points and channel combinations. The box to the right of the phase difference tracings are the phase difference values (degrees) at exactly 3 minutes and 4 seconds from the start of the recording. This part of the EEG recording was artifact free. A careful comparison demonstrates that the phase differences have been altered in each and every artifact free time sample for the entire EEG record and for all frequencies and all channel combinations.

recording (see National Library of Medicine Pubmed search using EEG as the search term). Mathematical decomposition of the EEG without replacement of an altered time series was introduced in the $1960 \mathrm{~s}$ $[43,44]$. For example, principal components analysis decomposes EEG features that are correlated and varimax rotation to separate loadings on orthogonal axes. Independent Component Analysis (ICA) was introduced in the 1990s [42,34] and differs from principal components analysis by decomposing a multivariate signal into independent nonGaussian components. ICA is a special case of blind source separation such as in the "cocktail party problem" of listening in on one person's speech in a noisy room. ICA is a very useful decomposition method but lacks scientific validity when it comes to removing ICs and the replacement of the original EEG by an "artifact" that separates measurement from the ground truth of the electrogenesis of the EEG and the invertible physics of electrostatics described by Gauss, Laplace, Poisson, Green, Maxwell and von Helmholtz. A search of the National Library of Medicine (Pubmed) using the search terms "EEG test retest reliability" produces over 3,000 citations since the 1970s with quantitative EEG. To the best of our knowledge none of these citations used ICA replacement and all of them used the artifact deletion method with $\mathrm{P}>0.9$ test re-test reliabilities. Given this history there is no justification for the replacement of the artifact free parts of an EEG recording with artifact by ICA-R replacement when simple deletion of artifact by deletion is the historic standard.
Unfortunately, there are a growing number of EEG connectivity publications where phase differences and/or coherence were calculated after ICA-R replacement. The problem is a lack of validity because the non-invertible alteration of phase differences are not replicable and the results are not accurate measures of brain connectivity because the original physiologically dependent phase differences have been arbitrarily and irreversibly altered. We have found twenty exemplar publications that computed scalp EEG phase differences after ICA reconstruction/replacement and we are listing them here as a word of caution to note that the findings in these studies lack validity and reliability [45-65].

We recommend that the authors of these studies re-visit their raw data using standard artifact free selection methods and not ICA-R (a replacement of the artifact free parts of the original EEG) and then recalculate the EEG connectivity measures based on the invertible physics selections without any phase distortion. Then compare their results to the ICA-R dependent results. We also recommend that a standard for reviewers of EEG studies that used ICA-R to ask the authors to compare their results with and without the use of ICA-R replacement and if there are differences then to ask the authors to explain the validity and reliability of the differences. Finally, for legal and ethical purposes it is important to not replace a patient's artifact free parts of their EEG medical record with a distorted digital time series without the patient's knowledge and consent. 
Montage: LinkEars

\section{FFT Phase Lag Percent Difference (\%)}

Intrahemispheric: LEFT

\begin{tabular}{|c|c|c|c|c|}
\hline & DELTA & THETA & ALPHA & BETA \\
\hline $\mathrm{FP} 1 \mathrm{~F} 3$ & 10.61 & 96.82 & 36.86 & 76.19 \\
\hline $\mathrm{FP} 1 \mathrm{C}_{3}$ & 97.68 & 94.81 & 78.16 & 98.63 \\
\hline FP1 $P_{3}$ & 94.98 & 88.71 & 69.21 & 94.45 \\
\hline FP1 O1 & 82.75 & 68.22 & -2.59 & 23.59 \\
\hline FP1 F7 & 60.43 & 87.36 & -80.77 & 89.89 \\
\hline FP1 $T 3$ & 98.17 & 92.95 & -07.22 & 79.97 \\
\hline FP1 TS & 90.97 & 87.02 & -0.81 & 84.72 \\
\hline $\mathrm{F}_{3} \mathrm{C}_{3}$ & 70.02 & 40.58 & 27.79 & 77.38 \\
\hline $\mathrm{F} 3 \mathrm{P} 3$ & 90.52 & 84.27 & 84.03 & 95.52 \\
\hline F3 O1 & 89.82 & 72.45 & 1.52 & 56.98 \\
\hline $\mathrm{F} 3 \mathrm{F7}$ & 87.91 & 98.01 & 63.88 & 72.52 \\
\hline $\mathrm{F} 3 \mathrm{~T} 3$ & 97.84 & 99.88 & 52.63 & 98.08 \\
\hline F3 T5 & 99.40 & 87.88 & 74.79 & 85.03 \\
\hline $\mathrm{C}_{3} \mathrm{P}_{3}$ & 82.55 & 42.13 & 69.37 & 69.38 \\
\hline $\mathrm{C}_{3} \mathrm{O}_{1}$ & 92.41 & 80.91 & 88.77 & 88.85 \\
\hline $\mathrm{C}_{3} \mathrm{~F} 7$ & 97.30 & 85.38 & 51.53 & 96.64 \\
\hline $\mathrm{C}_{3} \mathrm{~T}_{3}$ & 83.93 & 73.95 & 19.19 & 48.16 \\
\hline C3 T5 & 98.06 & 73.81 & 92.65 & 90.77 \\
\hline P3 01 & 20.01 & 32.40 & 47.51 & -97.19 \\
\hline P3 F7 & 90.45 & 83.98 & 79.95 & 93.31 \\
\hline P3 T3 & 89.43 & 87.04 & 95.05 & 97.42 \\
\hline P3 TS & 47.28 & 47.28 & 93.78 & 14.10 \\
\hline $01 F 7$ & 83.00 & 57.01 & -1.30 & 23.44 \\
\hline $01 \mathrm{~T} 3$ & 78.84 & 89.74 & 71.07 & 86.72 \\
\hline $01 \mathrm{TS}$ & -8.46 & -52.05 & 73.32 & -93.86 \\
\hline F7 $\quad \mathrm{T} 3$ & 93.54 & 85.49 & -8.51 & 98.27 \\
\hline F7 T5 & 98.68 & -25.38 & 23.77 & 82.12 \\
\hline T3 T5 & 65.68 & -83.55 & 40.19 & -13.45 \\
\hline
\end{tabular}

Intrahemispheric: RIGHT

\begin{tabular}{|c|c|c|c|c|}
\hline & DELTA & THETA. & ALPHA & BETA \\
\hline $\mathrm{FP} 2 \mathrm{~F} 4$ & 13.67 & 83.35 & 77.89 & 99.28 \\
\hline $\mathrm{FP} 2 \mathrm{C} 4$ & 97.63 & 89.77 & 82.80 & 91.67 \\
\hline FP2 $P 4$ & 96.16 & 82.78 & 36.86 & 82.46 \\
\hline $\mathrm{FP} 2 \mathrm{O} 2$ & 88.90 & 62.10 & -1.14 & 7.04 \\
\hline FP2 F8 & 19.37 & -29.59 & 8.82 & -58.27 \\
\hline FP2 $\mathrm{T} 4$ & 97.55 & 73.23 & -95.20 & 99.07 \\
\hline FP2 TO & 95.55 & 71.40 & 10.92 & 10.02 \\
\hline $\mathrm{F} 4 \mathrm{C} 4$ & 41.39 & 50.75 & 29.95 & 27.44 \\
\hline $\mathrm{F} 4 \mathrm{P} 4$ & 98.75 & 85.80 & 72.00 & 94.35 \\
\hline $\mathrm{F} 4 \mathrm{O} \quad \mathrm{O}$ & 93.95 & 72.68 & 2.99 & 69.61 \\
\hline $\mathrm{F} 4 \mathrm{F8}$ & 81.48 & 64.67 & 93.30 & 94.07 \\
\hline $\mathrm{F} 4 \mathrm{~T} 4$ & 93.38 & 93.18 & 9.98 & 98.88 \\
\hline $\mathrm{F} 4 \mathrm{~T} 8$ & 99.15 & 84.94 & 42.83 & 91.70 \\
\hline $\mathrm{C}_{4} \mathrm{P} 4$ & 70.87 & 43.10 & -81.75 & 39.17 \\
\hline $\mathrm{C}_{4} \mathrm{O}_{2}$ & 94.99 & 81.23 & 95.48 & 87.14 \\
\hline $\mathrm{C}_{4} \mathrm{FB}$ & 94.14 & 76.30 & 16.38 & 94.80 \\
\hline $\mathrm{C}_{4} \mathrm{~T}_{4}$ & 91.16 & 19.72 & 37.71 & 96.49 \\
\hline $\mathrm{C} 4 \mathrm{Te}$ & 79.96 & 67.76 & 46.75 & 63.52 \\
\hline $\mathrm{P} 4 \mathrm{O} 2$ & 13.29 & 37.32 & 50.47 & 9.17 \\
\hline P4 F8 & 97.42 & 89.58 & 75.72 & 95.47 \\
\hline $\mathrm{P} 4 \mathrm{~T} 4$ & 98.57 & 5.74 & -50.09 & 95.54 \\
\hline P4 TO & 4.50 & 24.30 & 22.42 & 35.04 \\
\hline $\mathrm{O} 2 \mathrm{~F} 8$ & 95.41 & 04.22 & 0.81 & 48.64 \\
\hline $\mathrm{O} 2 \mathrm{~T} 4$ & -41.00 & -1.13 & 91.15 & 89.07 \\
\hline O2 T6 & -54.97 & 7.24 & 20.82 & 83.99 \\
\hline F8 T4 & 90.00 & -3.63 & -77.70 & 93.00 \\
\hline F8 T8 & 97.48 & -88.13 & 82.47 & 98.57 \\
\hline T4 T 8 & 89.18 & -27.03 & .71 .69 & 94.65 \\
\hline
\end{tabular}

Interhemispheric: HOMOLOGOUS PAIRS

\begin{tabular}{|c|c|c|c|c|}
\hline & DELTA & THETA & ALPHA & BETA \\
\hline FP1 FP2 & 90.31 & 75.53 & 0.59 & -94.00 \\
\hline $\mathrm{C}_{3} \mathrm{C}_{4}$ & 99.00 & 91.14 & 90.27 & 70.95 \\
\hline 0102 & 31.77 & -41.19 & 37.75 & 47.47 \\
\hline T3 T4 & 52.81 & -20.14 & 18.41 & 89.01 \\
\hline
\end{tabular}

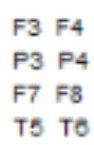

\begin{tabular}{|r|r|r|r|}
\multicolumn{1}{c}{ DELTA } & \multicolumn{1}{c}{ THETA } & \multicolumn{1}{l}{ ALPHA } & \multicolumn{1}{l}{ BETA } \\
\hline 68.09 & 53.52 & 2.47 & 27.62 \\
\hline 01.12 & 90.95 & 99.80 & 85.17 \\
\hline 97.52 & 55.03 & 15.99 & 51.03 \\
\hline-60.40 & 89.84 & 99.29 & 85.03 \\
\hline
\end{tabular}

Figure 7: The rows are channel pairs for the left and right hemisphere. The columns are percent difference of the phase values in the delta, theta, alpha and beta frequency bands. FFT Percent difference between 1 min \& 42 seconds of artifact free EEG in the original time series vs the ICA reconstructed time series using the same time points of $1 \mathrm{~min} \& 42 \mathrm{sec}$. The smallest difference was $0.59 \%$ for Fp1-Fp2 in the alpha band and the largest difference was $99.8 \%$ for P3-P4 in the alpha band.

\section{Invalidation of the physics of the EEG}

The reason that ICA invalidates the physics of electrostatics is because it replaces the original electrical potential measures with noninvertible substitutes of the original electrical potentials and distorts phase differences. For example, a charge $\mathrm{q}$ that moves a distance $\mathrm{dx}$ where the differences in electrical potential between the initial and final location is $\mathrm{dV}$, then the change in potential $\mathrm{d}=\mathrm{q} \mathrm{dV}$ and the work $\mathrm{W}$ used to move the charge is $\mathrm{W}=-\mathrm{qE} \mathrm{dx} \cos \theta$ where $\mathrm{E}$ is the local electrical field strength and $\theta$ is the angle between the direction of the field and the $\mathrm{x}$-axis [48]. If one replaces the original electrical potential in volts with an irretrievably altered value (ICA-R), then the change in the charge $\mathrm{q}$ and electrical potential is irretrievably invalid where one uses 1 or 18 ICs to create a non-invertible 19 channel alternate substitute reality or $\mathrm{dP} \neq \mathrm{dP}$ which is the case with ICA-R. These conclusions were tested in the tables and figures of this study and are consistent with the publications, simulations, mathematics and experts cited previously.

\section{Invalid justifications for ICA-R replacement of the original EEG recording}

An important issue is the justification for the ICA-R distortion by 
Montage: LinkEars

Pre-ICA.nga - Post-ICA.nga

FFT Phase Lag Paired t-Test (P-Value)

Intrahemispheric: LEFT

\begin{tabular}{|c|c|c|c|c|}
\hline & DELTA & THETA & ALPHA & BETA \\
\hline $\mathrm{FP} 1 \mathrm{~F} 3$ & 0.000 & 0.000 & 0.000 & 0.000 \\
\hline $\mathrm{FP} 1 \mathrm{C} 3$ & 0.000 & 0.000 & 0.000 & 0.000 \\
\hline FP1 P3 & 0.000 & 0.000 & 0.000 & 0.000 \\
\hline FP1 01 & 0.000 & 0.000 & 0.004 & 0.000 \\
\hline FP1 F7 & 0.000 & 0.000 & 0.060 & 0.000 \\
\hline FP1 13 & 0.000 & 0.000 & 0.000 & 0.000 \\
\hline FP1 T5 & 0.000 & 0.000 & 0.000 & 0.000 \\
\hline $\mathrm{F} 3 \mathrm{C} 3$ & 0.000 & 0.000 & 0.000 & 0.000 \\
\hline F3 P3 & 0.000 & 0.000 & 0.000 & 0.000 \\
\hline F3 01 & 0.000 & 0.000 & 0.000 & 0.000 \\
\hline F3 F7 & 0.000 & 0.000 & 0.000 & 0.000 \\
\hline F3 T3 & 0.000 & 0.000 & 0.000 & 0.000 \\
\hline F3 T5 & 0.000 & 0.000 & 0.000 & 0.000 \\
\hline C3 P3 & 0.000 & 0.000 & 0.000 & 0.000 \\
\hline C3 01 & 0.000 & 0.000 & 0.000 & 0.000 \\
\hline $\mathrm{C} 3 \mathrm{~F} 7$ & 0.000 & 0.000 & 0.000 & 0.000 \\
\hline C3 T3 & 0.000 & 0.000 & 0.000 & 0.000 \\
\hline C3 T5 & 0.000 & 0.000 & 0.000 & 0.000 \\
\hline P3 01 & 0.000 & 0.000 & 0.000 & 0.000 \\
\hline P3 F7 & 0.000 & 0.000 & 0.000 & 0.000 \\
\hline P3 T3 & 0.000 & 0.000 & 0.000 & 0.000 \\
\hline P3 T5 & 0.000 & 0.000 & 0.000 & 0.000 \\
\hline O1 F7 & 0.000 & 0.000 & 0.008 & 0.000 \\
\hline $01 \mathrm{~T} 3$ & 0.000 & 0.000 & 0.000 & 0.000 \\
\hline $01 \mathrm{T5}$ & 0.000 & 0.000 & 0.000 & 0.000 \\
\hline F7 $\quad \mathrm{T} 3$ & 0.000 & 0.262 & 0.955 & 0.000 \\
\hline F7 T5 & 0.000 & 0.000 & 0.148 & 0.000 \\
\hline T3 T5 & 0.000 & 0.012 & 0.003 & 0.000 \\
\hline
\end{tabular}

Intrahemispheric: RIGHT

\begin{tabular}{|c|c|c|c|c|}
\hline & DELTA & THETA & ALPHA & BETA \\
\hline $\mathrm{FP} 2 \mathrm{~F} 4$ & 0.000 & 0.000 & 0.000 & 0.000 \\
\hline $\mathrm{FP} 2 \mathrm{C}_{4}$ & 0.000 & 0.000 & 0.000 & 0.000 \\
\hline $\mathrm{FP} 2 \mathrm{P} 4$ & 0.000 & 0.000 & 0.000 & 0.000 \\
\hline $\mathrm{FP} 2 \mathrm{O} 2$ & 0.000 & 0.000 & 0.000 & 0.000 \\
\hline FP2 F8 & 0.000 & 0.000 & 0.003 & 0.347 \\
\hline $\mathrm{FP} 2 \quad \mathrm{~T} 4$ & 0.000 & 0.000 & 0.040 & 0.000 \\
\hline $\mathrm{FP} 2 \mathrm{~T} 8$ & 0.000 & 0.000 & 0.000 & 0.001 \\
\hline $\mathrm{F} 4 \mathrm{C} 4$ & 0.000 & 0.000 & 0.000 & 0.000 \\
\hline F4 P4 & 0.000 & 0.000 & 0.000 & 0.000 \\
\hline $\mathrm{F} 4 \mathrm{O} 2$ & 0.000 & 0.000 & 0.000 & 0.000 \\
\hline F4 F8 & 0.000 & 0.000 & 0.000 & 0.000 \\
\hline $\mathrm{F} 4 \mathrm{~T} 4$ & 0.000 & 0.000 & 0.000 & 0.000 \\
\hline $\mathrm{F} 4 \mathrm{~T} 6$ & 0.000 & 0.000 & 0.000 & 0.000 \\
\hline $\mathrm{C} 4 \mathrm{P} 4$ & 0.000 & 0.000 & 0.000 & 0.000 \\
\hline $\mathrm{C} 4 \mathrm{O} 2$ & 0.000 & 0.000 & 0.000 & 0.000 \\
\hline C4 F8 & 0.000 & 0.000 & 0.000 & 0.000 \\
\hline C4 T4 & 0.000 & 0.000 & 0.000 & 0.000 \\
\hline $\mathrm{C} 4 \mathrm{~T} 8$ & 0.000 & 0.000 & 0.000 & 0.000 \\
\hline $\mathrm{P} 4 \mathrm{O} 2$ & 0.001 & 0.000 & 0.000 & 0.001 \\
\hline P4 F8 & 0.000 & 0.000 & 0.000 & 0.000 \\
\hline P4 T4 & 0.000 & 0.000 & 0.000 & 0.000 \\
\hline P4 T6 & 0.005 & 0.000 & 0.000 & 0.000 \\
\hline $\mathrm{O} 2 \mathrm{~F} 8$ & 0.000 & 0.000 & 0.002 & 0.000 \\
\hline $\mathrm{O} 2 \mathrm{~T} 4$ & 0.000 & 0.000 & 0.000 & 0.000 \\
\hline O2 T6 & 0.003 & 0.019 & 0.000 & 0.003 \\
\hline F8 $\quad$ T4 & 0.049 & 0.174 & 0.954 & 0.000 \\
\hline F8 T6 & 0.000 & 0.008 & 0.155 & 0.000 \\
\hline T4 T6 & 0.002 & 0.005 & 0.000 & 0.000 \\
\hline
\end{tabular}

Interhemispheric: HOMOLOGOUS PAIRS

\begin{tabular}{|c|c|c|c|c|}
\hline & DELTA & THETA & ALPHA & BETA \\
\hline $1 \mathrm{FP} 2$ & 0.015 & 0.000 & 0.042 & 0.012 \\
\hline $\mathrm{C} 4$ & 0.000 & 0.000 & 0.000 & 0.000 \\
\hline $01 \mathrm{O}_{2}$ & 0.010 & 0.001 & 0.000 & 0.000 \\
\hline T4 & 0.000 & 0.000 & 0.567 & 0.000 \\
\hline
\end{tabular}

$$
\begin{array}{ll}
\text { F3 } & \text { F4 } \\
\text { P3 } & \text { P4 } \\
\text { F7 } & \text { F8 } \\
\text { T5 } & \text { T6 }
\end{array}
$$

\begin{tabular}{|r|r|r|r|}
\multicolumn{1}{c}{ DELTA } & \multicolumn{1}{c}{ THETA } & \multicolumn{1}{c|}{ ALPHA } & \multicolumn{1}{c|}{ BETA } \\
\hline 0.000 & 0.000 & 0.000 & 0.000 \\
\hline 0.000 & 0.000 & 0.000 & 0.000 \\
\hline 0.000 & 0.000 & 0.000 & 0.000 \\
\hline 0.000 & 0.000 & 0.000 & 0.000 \\
\hline
\end{tabular}

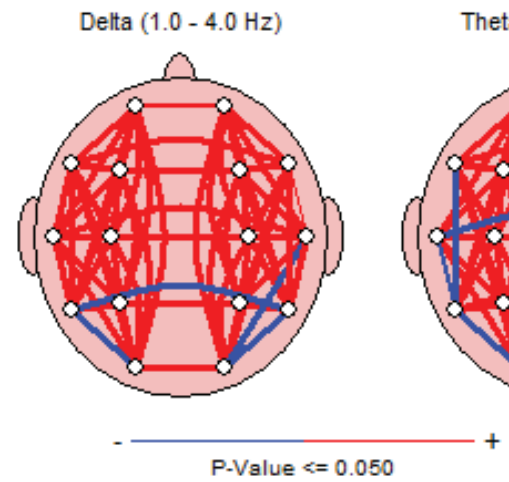

(C) 2001-2017, Applied NeuroScience, Inc

Theta $(4.0-8.0 \mathrm{~Hz})$

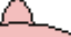

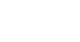
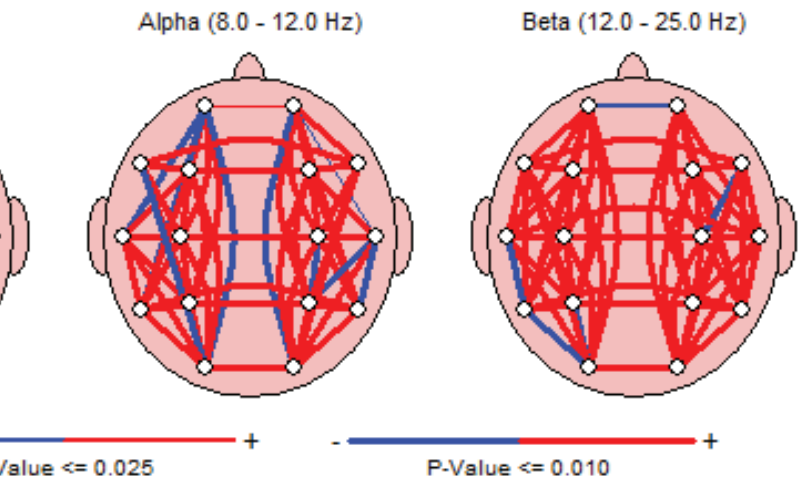

P-Value $<=0.010$

18

Figure 8: The rows are channel pairs for the left and right hemisphere. The columns are paired t-test results for the delta, theta, alpha and beta frequency bands between $1 \mathrm{~min} \& 42$ seconds of artifact free EEG in the original time series vs the ICA reconstructed time series using the same time points of $1 \mathrm{~min} \& 42 \mathrm{sec}$. A total of $256 \mathrm{t}$-tests were conducted and $96.5 \%$ were statistically significant. 
Table 1: Percentage of statistically significant $(P<0.05)$ ICA-R changes in phase difference between the original EEG and the ICA-R replacement of the original EEG. Subject 11 is the UCSD Eeglablist subject.

\begin{tabular}{|c|c|c|c|c|c|}
\hline T-TESTS & Overall & Delta & Theta & Alpha & Beta \\
\hline Subject 1 & $54.8 \%$ & $39.8 \%$ & $67.8 \%$ & $55.0 \%$ & $56.7 \%$ \\
\hline Subject 2 & $95.5 \%$ & $97.7 \%$ & $98.2 \%$ & $87.7 \%$ & $98.2 \%$ \\
\hline Subject 3 & $34.5 \%$ & $21.6 \%$ & $42.1 \%$ & $20.5 \%$ & $53.8 \%$ \\
\hline Subject 4 & $19.2 \%$ & $18.1 \%$ & $13.5 \%$ & $20.5 \%$ & $24.6 \%$ \\
\hline Subject 5 & $25.7 \%$ & $34.5 \%$ & $23.4 \%$ & $19.3 \%$ & $25.7 \%$ \\
\hline Subject 6 & $95.8 \%$ & $96.5 \%$ & $95.3 \%$ & $94.7 \%$ & $96.5 \%$ \\
\hline Subject 7 & $45.3 \%$ & $28.1 \%$ & $62.6 \%$ & $33.3 \%$ & $57.3 \%$ \\
\hline Subject 8 & $64.6 \%$ & $53.8 \%$ & $76.0 \%$ & $60.8 \%$ & $67.8 \%$ \\
\hline Subject 9 & $33.9 \%$ & $21.6 \%$ & $33.9 \%$ & $14.0 \%$ & $66.1 \%$ \\
\hline Subject 10 & $75.9 \%$ & $80.7 \%$ & $80.1 \%$ & $78.4 \%$ & $64.3 \%$ \\
\hline Subject 11 & $52.9 \%$ & $54.4 \%$ & $59.6 \%$ & $41.5 \%$ & $56.1 \%$ \\
\hline \% Average & $54.4 \%$ & $49.7 \%$ & $59.3 \%$ & $47.8 \%$ & $60.7 \%$ \\
\hline
\end{tabular}

a non-invertible ICA replacement of the original electrical physics of the human EEG with a mathematical construct that is physiologically and mathematically invalid. There are two frequently used invalid justifications:

1- EEG is all noise to begin with and therefore an alternate ICA universe is of equivalent value, or

2- $\quad$ There is no physiological ground truth of EEG and phase differences in the first place and hence no harm in replacing the original EEG recording. There are discussions about the concept of "non-stationarity" and high density 128 channels $v s .19$ channels and the relative magnitude of "corruption" but these are academic without recognition that there must be zero tolerance of distortion at the primary level of recording of the physics of volts and time no matter how many recording sensors were used.

\section{Acknowledgements}

We want to thank Arnaud Delorme and others on the UCSD Eeglablist for their comments and dialog and careful ICA analyses of the Mitsar EEG data sample. This study was support by USDA grant (CRS 801-12-09C) and NIH (RR 08079 09). This study was exempt from the requirement of review by an ethics committee because only post hoc statistical analyses on pre-existing data stored in archives from patients who signed a consent form was used and all patient identifiers have been removed.

\section{Declaration of Conflicting Interests}

The Author(s) declare that there is no conflict of interest.

\section{References}

1. Berger H (1929) Uber das Elektrenkephalogramm des Menschen. Archieves Psychiatric Nervenkrankh 87: 527-570.

2. Berger H (1932) Uber das Electrenkephalogramm des Menschen. Vierte Mitteilungj. Archiv Fur Psychiatrie und Neverkrankheiten 97: 6-26.

3. Berger H (1934) Uber das Electrenkephalogramm des Menschen. Neunte Mitteilungj. Archiv. Fur. Psychiatrie und Neverkrankheiten 102: 538-557.
4. Niedermeyer E, Lopes da Silva F (2005) Electroencephalograph: Basic Principles, Clinical Applications and Related Fields, Wilkins and Williamson, Baltimore, USA.

5. Cooper R, Winter AL, Crow Hj, Walter WG (1965) Comparison of subcortical, cortical, and scalp activity using chronically indwelling electrodes in man. Electroencephalogr Clin Neurophysiol 18: 217228.

6. Cooper R, Osselton JW, Shaw JC (1974) EEG Technology. Butterworth $\&$ Co, London.

7. Cooper $R$ (1975) Measurement of time and phase relationships of the EEG. In: Dolce G, Kunkel H (Eds.) Computerized EEG Analysis, Gustav Fischer Verlag, Stuttgart.

8. Adey WR, Walter DO, Hendrix CE (1961) Computer techniques in correlation and spectral analyses of cerebral slow waves during discriminative behavior. Exp Neurol 3: 501-524.

9. Adey WR, Walter DO (1963) Application of phase detection and averaging techniques in computer analysis of EEG records in the cat. Exper Neurol 7: 186-209.

10. Adey WR (1964) Data acquisition and analysis techniques in a Brain Research Institute. Ann N Y Acad Sci 115: 844-866.

11. Adey WR (1964) Biological instrumentation. electrophysiological recording and analytic techniques. Physiologist 72: 65-68.

12. John ER (1977) Functional Neuroscience, Neurometrics: Quantitative Electrophysiological Analyses, L. Erlbaum Associates.

13. Duffy F, Hughes JR, Miranda F, Bernad P, Cook P (1994) Status of quantitative EEG (QEEG) in clinical practice. Clin Electroencephalography 25: VI-XXII.

14. Freeman WJ, Rogers LJ (2002) Fine temporal resolution of analytic phase reveals episodic synchronization by state transitions in gamma EEGs. J Neurophysiol 87: 937-945.

15. Freeman WJ, Burke BC, Homes MD (2003) Aperiodic phase resetting in scalp EEG of beta-gamma oscillations by state transitions at alpha-theta rates. Hum Brain Mapp 19: 248-272.

16. Freeman WJ, Homes MD, West GA, Vanhatlo S (2006) Fine spatiotemporal structure of phase in human intracranial EEG. Clin Neurophysiol 117: 1228-1243. 
17. Freeman WJ (1975) Mass action in the nervous system. Academic Press, NY.

18. Nunez PL, Srinivasan R (1981) Electrical Fields of the Brain. Oxford Scholarship Online, New York.

19. Nunez PL (1995) Neocortical Dynamics and Human EEG Rhythms. $1^{\text {st }}$ Edition, Oxford University Press, New York.

20. Thatcher RW, Krause P, Hrybyk M (1986) Corticocortical Association Fibers and EEG Coherence: A Two Compartmental Model. Electroencephalog. Clin Neurophysiol 64: 123-143.

21. Thatcher RW (2016) Handbook of Quantitative Electroencephalogreaphy and EEG Biofeedback. Anipublishing Inc, St. Petersburg, $\mathrm{Fl}$.

22. Aydorea S, Pantazisb D, Leahy RM (2013) A Note on the Phase Locking Value and its Properties. Neuroimage 74: 231-244.

23. Meng L, Frei MG, Osorio I, Strang G, Nguyen TQ (2004) Gaussian mixture models of ECoG signal features for improved detection of epileptic seizures. Med Eng Phys 26: 379-393.

24. Zhang R, Xu P, Guo L, Zhang Y, Li P, et al. (2013) Z-Score Linear Discriminant Analysis for EEG Based Brain-Computer Interfaces. PLoS ONE 8: e74433.

25. Hyvarinen A, Karhunen J, Oja E (2001) Independent Components Analysis. John Wiley \& Sons, NY.

26. Jervis BW, Coelho, M, Morgan G (1989) Effect on EEG responses of removing ocular artifacts by proportional EOG subtraction. Med BiolEng Comput 27: 484-490.

27. Montefusco-Siegmund R, Maldonado, PE, Devia C (2013) Effects of ocular artifact removal through ICA decomposition on EEG phase. $6^{\text {th }}$ Annual International IEEE EMBS Conference on Neural Engineering San Diego, California.

28. Hughes JR, John ER (1999) Conventional and quantitative electroencephalography in psychiatry. J Neuropsychiatry Clin Neurosci 11: 190-208.

29. Thatcher RW (2012) Coherence, Phase Differences, Phase Shift, and Phase Lock in EEG/ERP Analyses. Dev Neuropsychol 37: 476-496.

30. Delorme A, Makeig S (2004) EEGLAB: an open source toolbox for analysis of single-trial EEG dynamics including independent component analysis. J Neurosci Methods 134: 9-21.

31. Delorme A, Sejnowski T, Makeig S (2007) Enhanced detection of artifacts in EEG data using higher-order statistics and independent component analysis. Neurolmage 34: 1443-1449.

32. Jung T, Makeig S, Humphries C, Lee T, McKeown M, et al. (2000) Removing electroencephalographic artifacts by blind source separation. Psychophysiol 37:163-178.

33. Thatcher RW, North D, Biver C (2008) Intelligence and EEG phase reset: A two-compartmental model of phase shift and lock. Neurolmage 42: 1639-1653.

34. Thatcher RW, North D, Biver C (2009) Self organized criticality and the development of EEG phase reset. Human Brain Mapp 30: 553 574.

35. Bendat JS, Piersol AG (1980) Engineering applications of correlation and spectral analysis. Wiley, New York.

36. Mannan MMN, Jeong MY, Kamran MA (2016) Hybrid ICA-Regression: Automatic Identification and Removal of Ocular Artifacts from Electroencephalographic Signals. Front Hum Neurosci 10: 193.
37. Stone JV (2002) Independent component analysis: an introduction. Trends Cogn Sci 6: 59-64.

38. Castellanos NP, Makarov VA (2006) Recovering EEG brain signals: artifact suppression with wavelet enhanced independent component analysis. J Neurosci Methods 158: 300-312.

39. John ER, Ruchkin DS, Villegas J (1964) Signal analysis and behavioral correlates of evoked potential configurations in cats. Ann NY Acad Sci 112: 362-420.

40. Donchin E (1966) A multivariate approach to the analysis of average evoked potentials. IEEE Trans Biomed Eng 13: 131-139.

41. Pierre C (1994) Independent Component Analysis: a new concept? Signal Processing 36: 287-314.

42. Bell AJ, Sejnowski TJ (1995) An information-maximization approach to blind separation and blind deconvolution. Neural Comput 7 : 1129-1159.

43. de Pasquale F, Della Penna S, Sporns O, Romani GL, Corbetta M (2015) A dynamic core network and global efficiency in the resting human brain. Cerebral Cortex 26: 4015-4033.

44. Lai M, Demuru M, Hillebrand A, Fraschini M (2017) A Comparison Between Scalp-And Source-Reconstructed EEG Networks. Sci Rep 8 12269.

45. Colclough GL, Woolrich MW, Tewarie PK, Brookes MJ, Quinn AJ, et al. (2016) How reliable are MEG resting-state connectivity metrics? Neurolmage 138: 284-293.

46. Kuntzelman K, Miskovic V (2017) Reliability of graph metrics derived from resting state human EEG. Psychophysiol 54: 51-61.

47. Siems M, Pape AA, Hipp JF, Siegel M (2016) Measuring the cortical correlation structure of spontaneous oscillatory activity with EEG and MEG. Neurolmage 129: 345-355.

48. Toppi J, Astolfi L, Poudel GR, Innes CR, Babiloni F, et al. (2016) Timevarying effective connectivity of the cortical neuroelectric activity associated with behavioural microsleeps. Neuroimage 124: 421-432.

49. Farahibozorg SR, Henson RN, Hauk O (2017) Adaptive Cortical Parcellations for Source Reconstructed EEG/MEG Connectomes. Neuroimage 169: 23-45.

50. Rueda-Delgado LM, Solesio-Jofre E, Mantini, D, Dupont P, Daffertshofer A, et al. (2016) Coordinative task difficulty and behavioural errors are associated with increased long-range beta band synchronization. Neurolmage 146: 883-893.

51. Cooper PS, Wong AS, Fulham WR, Thienel R, Mansfield E, et al. (2015) Theta frontoparietal connectivity associated with proactive and reactive cognitive control processes. Neuroimage 108: 354-363.

52. Nayak CS, Bhowmik A, Prasad PD, Pati S, Choudhury KK, et al. (2017) Phase Synchronization Analysis of Natural Wake and Sleep States in Healthy Individuals Using a Novel Ensemble Phase Synchronization Measure. J ClinNeurophysiol 34: 77-83.

53. Vecchio F, Miraglia F, Piludu F, Granata G, Romanello R, et al. (2017) "Small World" architecture in brain connectivity and hippocampal volume in Alzheimer's disease: a study via graph theory from EEG data. Brain imaging and behavior 11: 473-485

54. Ranzi P, Freund JA, Thiel CM, Herrmann CS (2016) Encephalography Connectivity on Sources in Male Nonsmokers after Nicotine Administration during the Resting State. Neuropsychobiol 74: 48-59.

55. Vecchio F, Miraglia F, Curcio G, Della Marca G, Vollono C, et al. (2015) Cortical connectivity in fronto-temporal focal epilepsy from EEG analysis: a study via graph theory. Clin Neurophysiol 126: 11081116. 
56. Smit DJ, de Geus EJ, Boersma M, Boomsma DI, Stam CJ (2016) Lifespan development of brain network integration assessed with phase lag index connectivity and minimum spanning tree graphs. Brain connect 6: 312-325.

57. Jae WC, Ofori E, Misra G, Hess WC, Vaillancourt DE (2017) Betaband activity and connectivity in sensorimotor and parietal cortex are important for accurate motor performance. Neurolmage 144: 164-173.

58. Shou G, Ding L (2015) Detection of EEG spatial-spectral-temporal signatures of errors: A comparative study of ICA-based and channelbased methods. Brain topography 28: 47-61.

59. Kline JE, Huang HJ, Snyder KL, Ferris DP (2016) Cortical Spectral Activity and Connectivity during Active and Viewed Arm and Leg Movement. Front neurosci 10.

60. van Driel J, Gunseli E, Meeter M, Olivers CLN (2017) Local and interregional alpha EEG dynamics dissociate between memory for search and memory for recognition. Neurolmage 149: 114-128.
61. Mehrkanoon S, Breakspear M, Britz J, Boonstra TW (2014) Intrinsic coupling modes in source-reconstructed electroencephalography. Brain connect 4: 812-825.

62. Jackson JD (1999) Classical Electrodynamics. $3^{\text {rd }}$ Edition. John Wiley \& Sons, USA.

63. Thatcher $R$ (2010) Validity and reliability of quantitative electroencephalography. J Neurother 14: 122-152.

64. Thatcher RW, Lubar JF (2008) History of the scientific standards of QEEG normative databases. In: Budzinsky TH, Evans BJ, Abarbanel A (eds) Introduction to QEEG and Neurofeedback: Advanced Theory and Applications. Academic Press, San Diego, CA.

65. Wallstrom G, Kass R, Miller A, Cohn J, Fox N (2004) Automatic correction of ocular artifacts in the EEG: a comparison of regressionbased and component-based methods. Int J Psychophysiol 53: 105119 